\title{
Enhancing quality of services using weight based dynamic buffer management in cluster wireless mobile sensor network
}

\author{
Eid Rehman*, Muhammad Sher, Syed Husnain Abass Naqvi, Kamran Ullah \\ Department of Computer Science and Software, International Islamic University, Islamabad, Pakistan
}

\section{A R T I C LE IN F O}

\section{Article history:}

Received 15 May 2017

Received in revised form

17 August 2017

Accepted 22 August 2017

\section{Keywords:}

Quality of services

Buffer queue

WMSN

Energy efficiency

\begin{abstract}
A B S T R A C T
In this paper a buffer management algorithm which efficiently mange's $\mathrm{CH}$ buffer queues using an active queue buffer management technique by allocating dynamic buffer size of $\mathrm{CH}$ buffer to all neighboring nodes in ratio to the number of received packets which control packet dropping possibilities has been proposed. Furthermore, to handle overfull for particular queue, efficient packet dropping scheme is presented which enhance packets deliveries by allocating weight measurements, alluded to as need priority reversal, in which packet of high weight with low need dropped later than those of the high priority packet with low weight. Simulation discloses that the proposed scheme is a way to improve the packet delivery ratio, decrease packets drop and some other important network.
\end{abstract}

(C) 2017 The Authors. Published by IASE. This is an open access article under the CC BY-NC-ND license (http://creativecommons.org/licenses/by-nc-nd/4.0/).

\section{Introduction}

With the rapid and historic advancement in communication technologies over the last two decades, the wireless sensor networks are matured enough as a capable tool for monitoring the physical world (Mitra and Nandy, 2012). These networks consist of hundreds or even thousands of autonomous micro devices called "motes" or simply sensor nodes with sensing, processing and communicating capabilities. A typical wireless sensor network consists of a collection of static, mobile or a mixture of static and mobile sensor nodes which can communicate with each other for exchanging data efficiently. The wireless sensor networks whose all or some sensors have the capability of movement around the deployed area are called Wireless Mobile Sensor Networks (WMSN) (Liu et al., 2007).

The WMSN are ideally used in commercial, civil and military applications for continuous event detection and location sensing. The sensor nodes are limited in terms of processing power, buffer storage space and most importantly the energy resources. Energy minimization and buffer management of a node are the major limitations in WMSN.

Researchers have put a lot of efforts to develop energy efficient clustering protocols in WMSN in

\footnotetext{
* Corresponding Author.

Email Address: eidrehmanktk@gmail.com (E. Rehman) https://doi.org/10.21833/ijaas.2017.010.016

2313-626X/C) 2017 The Authors. Published by IASE.

This is an open access article under the CC BY-NC-ND license

(http://creativecommons.org/licenses/by-nc-nd/4.0/)
}

order to enhance the lifetime and quality of services (QoS) of network. For this purpose the hierarchical (Al-Karaki and Kamal, 2004) protocol segregates the network nodes into sub sections called clusters. Each cluster consists of one representative node called cluster head $(\mathrm{CH})$ and other member nodes. The cluster member nodes are responsible for gathering information from the surroundings and forward this information toward the relative $\mathrm{CH}$ where redundant data is fused using data aggregation techniques or forward directly to the base station. However, excessive amount of data transmission and interference from neighbor nodes may cause network congestion at the $\mathrm{CH}$ (Jiang et al., 2009).

In MWSN, the packets are sends from one node to another node via $\mathrm{CH}$ which transmit packets to the destination. But, each node takes part instantaneously in numerous packets transmissions. $\mathrm{CH}$ also performs data aggregation on the received and as a result, $\mathrm{CH}$ may store different packets of member nodes. These packets needed to be communicated when channel becomes idle.

However, there are numerous types of packets i.e. real-time and non-real time. On the basis of application some critical packets needs instant transmission.

Initially $\mathrm{CH}$ assigns an equal size queue of its buffer to the member nodes like in Fig. 1. For QoS requirement, the $\mathrm{CH}$ buffer management and packet scheduling plays an important role in terms of number of packets that can be hold in allocated queue for specific node before this queue is full and the newly arrived packets are dropped. Similarly, packet scheduling assigns priorities to transmission 
streams with QoS considerations. Then packets are queued and scheduled according to the assigned priorities, which are related to the effective periods of event reports.

There are many variations introduced in the research of QoS paradigm about how these queues are managed and packet schedule at $\mathrm{CH}$. Normally drop tail and front end technique has been used for $\mathrm{CH}$ queue management which drops packet for front or tail of queue when the queue is full and no queue size extension is allowed in case of some member nodes allocated queue have free space. Later active queue management (AQM) (Periyasamy and Perumal, 2015) techniques have been developed, but they do not work well in dynamic network like WMSN. As WMSN is resources constrained environment, if some nodes behave aggressively by sending packets at higher data rate to the base station through $\mathrm{CH}$ then normal nodes observe unfair buffer queue occupancy. Mean if some nodes does not fully utilize there allocated queue, than how this free space will be assign to the other indigent node.

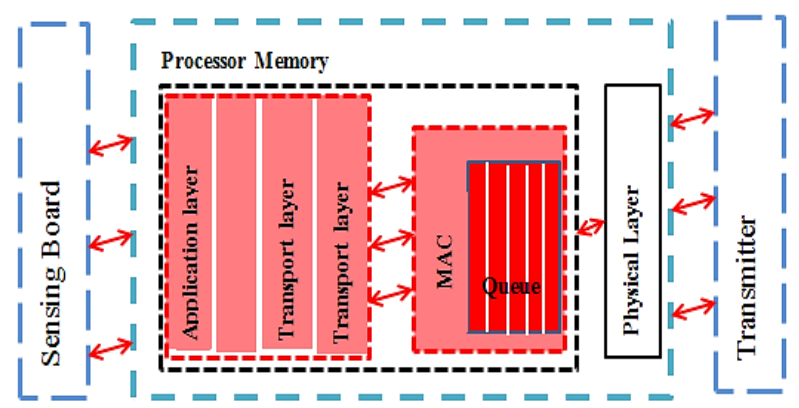

Fig. 1: Cluster head buffer queue allocation

For the instant, WMSN buffer management which is linked to traffic management basis on the weight is in a relatively early stage. Prioritization of the store packets in $\mathrm{CH}$, subsequently, has turn into a compelling issue, in light of the fact that a predetermined number of packets can be transmitted. A noticeable packet ought to have higher priority than other packets for delivery. This situation brings starvation to the some packets.

This paper presents queue management and packet scheduling algorithms for $\mathrm{CH}$ buffer to enhance life time and QoS. This algorithm efficiently mange's $\mathrm{CH}$ buffer using an active queue management strategy by allocation dynamic buffer size to all member nodes in fraction to the number of packets received from it. This process can control packet dropping probabilities. Also assigning packet dynamic weight is more appealing approaches to handle traffic adjustment what's more, give reasonable opportunities to all packets toward destination distant from the fixed priority's mechanism. The presented dropping algorithm enhances packet deliveries by allocating weight measurements, alluded to as priority reversal. In this mechanism packet of low priority having higher weight can be dropped late than those of the high priority packets having low weight value.
The remaining parts of the paper are organized as follows. Section 2 provides the literature review of some well-known $\mathrm{CH}$ buffer queue management algorithm for mobile WSNs. Section 3 describes buffer management algorithm. Section 4 describes proposed weight based dropping policy scheme. Section 6 presents the energy consumption model and section 7 discuss mobility model of our scheme. Section 7 describes the simulation results of our proposed scheme.

\section{Related works}

First come first serve (FCFS) algorithm (Vanithamani and Mahendran, 2014) operates packets are on the basis of arrival time. Thus $\mathrm{CH}$ need more waiting time for packets from different cluster sensor nodes than packets from local node. This needs additional time for transferring packets to destination node. Active Queue Management $(A Q M)$ is usually used to take care of buffer queues management and scheduling issues, which is implemented at source-node side. Random Early Detection (RED), as one of the most popular AQM schemes, keeps long queues proactively for alleviating network traffic problems in queue management (Floyd and Jacobson, 1993). Depending on the current queue length threshold, RED randomly drops packets to achieve high utilization. The RED algorithm is initially designed to analyze and forecast congestion at source-node side, instead of the whole network.

The authors (Nasser et al., 2013) propose a Dynamic Multilevel Priority (DMP) packet scheduling scheme for Wireless Sensor Networks (WSNs). The scheme uses three-level of priority queues to schedule data packets based on their types and priorities at (Lin et al., 2011) presents an approach that makes two major innovations in cluster WMSNs. First, DPCC utilizes dynamic priority to speak to packet significance. Second, it organizes the neighborhood activity of nodes close to the base station when WMSN is exceedingly congested.

The author (Li et al., 2013) proposed a dynamic queue management approach based on priority is needed in WMSN to process the urgent event and settle the problem how to send the data to the sink timely and efficiently. A novel cross-layer data queue management approach is presented to provide dynamic data queue management. In this paper $\mathrm{CHu}$ and Guo, 2016), the author has adopted the additiveincrease multiplicative-decrease (AIMD) scheme and proposed an improved RED algorithm by using neighbor feedback in cluster wireless sensor network (CWSM). A congestion control model in CWSNs has been designed, which is a linear system with a non-linear feedback modeled by a Lur'e type system, but does not does not consider the effect of mobility.

The author proposed (Justus and Sekar, 2016) packet scheduling algorithm called Enhanced dynamic priority (EDP) basis on delay and loss constraints. According to this scheme, each received 
packet is examined and prioritized in the view of delay and loss constraints. Then the prioritized packets are arranging according to packet priority which result starvation to the packets of medium and low priority classes.

Another proposed algorithm (Yantong and Sheng, 2016), there are three various types of queue in $\mathrm{CH}$. One is called higher priority queue where only realtime packets are stored, while non-real packets are stored in middle priority queue which will be transmitted to different node, and lower priority queue holds non-real packets which are transmitted locally. The scheduling sequence in higher priority is based on the priority of packets; still scheduling sequence in the other queues is FCFS.

Bansode and Sambare (2015) displayed that concerning node to-node transmission delay; DMP packet scheduler is beating the current FCFS and Multilevel Queue Scheduler. Their scheme may reduce overhead and enhance bandwidth utilization that they expel the information packets with lapsed due dates.

Similarly lot of works (Chipara et al., 2013; Gomathi and Mahendran, 2015; Tsai et al., 2010) have been done on queue management in WSN but most focus on static without considering the mobility of node and cluster structure.

From latest research in WMSN, it has been obviously verified that most of the schemes focus only on packet scheduling of $\mathrm{CH}$ buffer. To the best of our knowledge, no one has discussed how to utilize free space of $\mathrm{CH}$ buffer if their one node allocated queue space full. Furthermore the accurate buffer management and packet scheduling policy for $\mathrm{CH}$ provides an excellent strategy for improving efficiency in terms of energy and QoS in cluster WMSN.

To maximize the efficiency of WMSN, a weighted fair scheduling algorithm is required to pre-arrange the weight of the network parameters for each cluster. Each $\mathrm{CH}$ makes appropriate queue management and packet scheduling with high rate adjustment to realize global cluster traffic. The $\mathrm{CH}$ is able to dynamically adjust its queue size and packet scheduling based upon varying network conditions, especially some member nodes generates huge traffic in the same cluster, which is called as selfadjustment.

\section{Proposed scheme}

\subsection{Buffer queue extension management algorithm of cluster head}

Before given the detail of proposed scheme, we assumed that there is already formed cluster and $\mathrm{CH}$ selection is done using some suitable clustering algorithms in WMSN. Most of CH uses Drop tail or FCFS scheme for its buffer queue management. To the best of our knowledge, we are going to present new buffer queue algorithm for $\mathrm{CH}$ buffer management. For a Selected $\mathrm{CH}$ node, the buffer queue is sustained in such a way that an equal buffer space is allocated to each member node. Further extension in queue size of buffer is also available to every member nodes to avoid any under-utilization of resources. The allocation of $\mathrm{CH}$ buffer is made on the basis of number of received packets to efficiently utilize the buffer space without any control of some neighboring source. The selected $\mathrm{CH}$ should know about their neighboring nodes.

Assume, there is one $\mathrm{CH}$ surrounded by other twelve member nodes in cluster as shown in Fig. 2. As these nodes are mobile, so some nodes may leave cluster and some new nodes join this cluster. The $\mathrm{CH}$ node is analyzed in our algorithm as a queue management node, i.e. it is used to allocate buffer space to its member nodes according to our proposed scheme. The $\mathrm{CH}$ node allocates equal buffer space to all of its member nodes in the initialization phase of buffer allocation. If Tbs is total buffer space (Tbs) of $\mathrm{CH}$ and $\mathrm{N}$ is the neighboring member nodes than the allocated buffer space (Abs) for each member node is calculated as given in (Rehman et al., 2016) Eq. 1.

$A b s \frac{T b s}{N}$

Here consider the case, where some member node reaches its maximum allocated buffer queue space in $\mathrm{CH}$, but the space is left in the $\mathrm{CH}$ total buffer due to the reason that one or more other member nodes are not communicating to $\mathrm{CH}$, or some nodes leave the cluster due to high or less mobility. Therefore, if we restrict a node to only allocate buffer space but the space is still available in the $\mathrm{CH}$ buffer, there is a chance that buffer space is not fully utilized. So we provide an extension to each member node for instantaneous space left in the $\mathrm{CH}$ buffer.

If some node send more packets to $\mathrm{CH}$ and reached the assigned limit in the buffer Queue space of $\mathrm{CH}$, but portion of the buffer space of $\mathrm{CH}$ is still unoccupied because other member nodes are communicating with lesser number of packets than their assigned limit. Therefore, for an effective utilization of the remaining buffer space of $\mathrm{CH}$, we determine the residual buffer space "Rbs" and divide it amongst all member nodes in proportion to their existing occupied buffer spaces. This division is logical and it effectively reduces the allocated buffer space for nodes which communicate with lesser numbers of packets but increases the same for nodes which stand equal or closer to their assigned buffer space. To provide extend buffer space to those member nodes that generates more traffic and occupied their assigned buffer queue node using unoccupied buffer space of CH. The Eq. 2 (Rehman et al., 2016) used to provide extend buffer space to those nodes.

$E b s_{j}=\left|\frac{R b s}{\sum_{i=1}^{n}(i)}\right| * N$

Here i represent the index of the number series from one to the number of member nodes $j$ denote the $\mathrm{j}$-th member node. For better understanding, 
suppose $75 \backslash \%$ of buffer space of $\mathrm{CH}$ is occupied and $25 \backslash \%$ is currently unoccupied due to either some node communication with fewer packets or leave cluster due to mobility. The residual unoccupied buffer space .i.e. $25 \backslash \%$ is divided with a summation figure obtained by adding a number series from 1 to member node (4) in cluster like in Fig. 2. Therefore, $25 \backslash \%$ unoccupied space is divided by $45(1+2+3+4)$ and multiple the result with the largest number of the series which is 4 in Fig. 2. The largest number is multiplied, so that the largest share from residual buffer space can be allocated to the node having reached the assigned maximum limit. The floor value is taken from the result and it is added to the instantaneous buffer space occupied by that node.

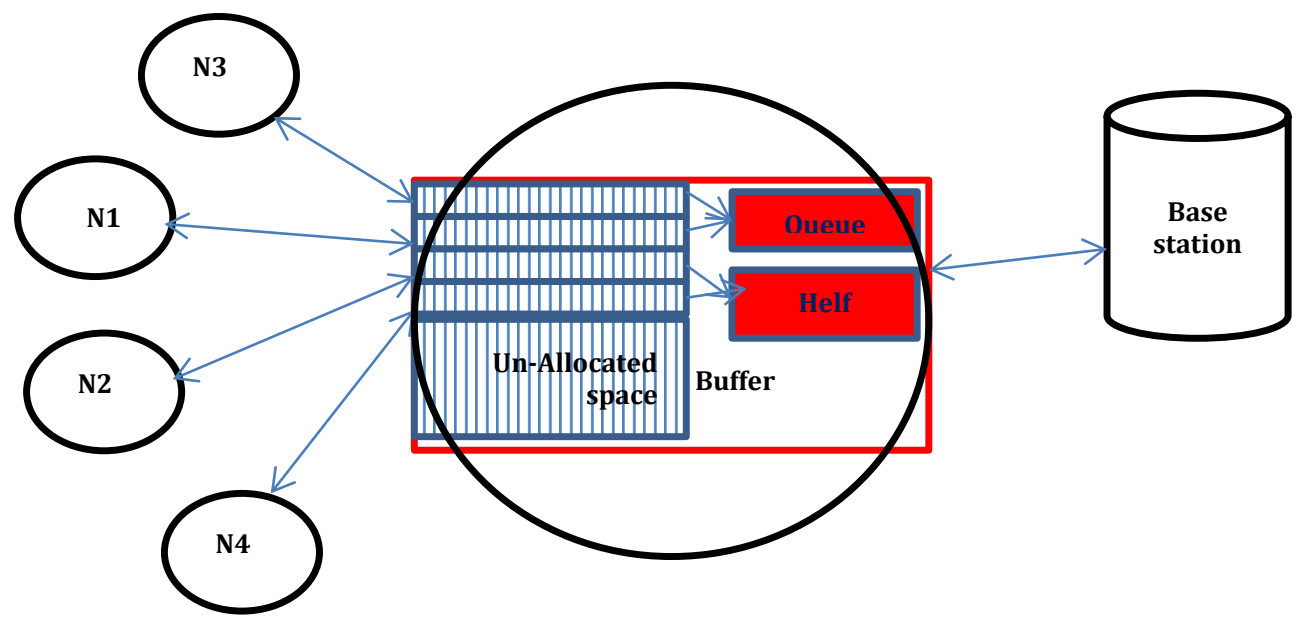

Fig. 2: Communications through $\mathrm{CH}$ in WMSN using $\mathrm{CH}$ buffer allocated and un-allocated space

Similarly, the member node that occupies the second largest assigned buffer space limit in $\mathrm{CH}$ would be assign second extend buffer spaces of $\mathrm{CH}$ using Eq. 3 (Rehman et al., 2016).

$E b s_{j-1}=\left|\frac{R b s}{\sum_{i=1}^{n}(i)}\right| *(N-1)$

Here i-1 means the second nodes that have reached to its maximum buffer queue allocated space in cluster. Similarly N-1 means that the result is multiple with second largest number of the series i.e 3 (4-1). This shows that the series $\mathrm{N}, \mathrm{N}-1, \mathrm{~N}-2,---$, 1 effectively contains normalization factors along with the sum of member nodes indies for the proposed scheme. The multiplication factor $\mathrm{N}$ of Eq. 2 is replaced by $\mathrm{N}-2$ for third node, $\mathrm{N}-3$ for Node 4 and so on.

The reason of dividing residual buffer space ( $\mathrm{Rbs}$ ) with a sum of member nodes indies and multiplying with components of the series of such indies is to allocate the higher calculated limits to the member nodes which are closer to assigned buffer space limits in proportion to the number of packets received. The packets dropping probabilities have been decrease by assigning dynamic buffer space to member nodes proportional to the number of packets received.

When new node joint the cluster and it is notified to $\mathrm{CH}$, the proposed scheme reconfigures assign buffer queue is such war that an equal queue is allocated to all member including new one. When the member node is move away from cluster than $\mathrm{CH}$ allocates equal share of the space to existing neighbors. The algorithm 1 shows the proposed scheme buffer queue management algorithm for $\mathrm{CH}$.
One question is that how much buffer extension should be allowed to a node? For this reason maximum and minimum buffer queue size thresholds are set for each node. The buffer queue size must be bounded by these threshold values. Active queue management's techniques (Shuaib and Aghvami, 2009) can also be applied in the proposed technique which notifies the member node when it's assigned space its limits in the $\mathrm{CH}$ buffer. The member node decreases the sending packets rates so that the current occupied queue space can be made sufficiently available again after processing or dequeuing packets. Further packets lost can be reduce by using AQM techniques because these techniques sent alert to reduce sending packets on the expected occurrence of reaching its assigned buffer space which provide accommodations to packets in the queue. The Table 1 shows how the $\mathrm{CH}$ buffer is divided into different queue initially and the receiving of packets in these queues for member nodes. Table 2 shows that how the queue management algorithm enables queue extension on those how received larger number of packet then allocated queue.

\subsection{Packet scheduling algorithm}

The algorithm 1 provides extension to member node allocated queue size at $\mathrm{CH}$ buffer. One problem is that when the member node allocated queue full and no further extension allowed in queue size to accommodate incoming packet than, the node refuses to store any more packets from its neighboring nodes, which may result to dropping packets. The previous works mostly used drop tail (FIFO) or drop front (LIFO) techniques to handle this issue in WMSN. Packet scheduling algorithm focuses 
on drop policy to drop or forward which new incoming packet. Some algorithms (Hu and Guo 2016; Li et al., 2013) used priority to drop packets by assigning priority to each packet and as a result, this brings starvation to the messages of medium and low priority classes. With a specific end goal to utilize every queue of the buffer efficiently, we use weight measurement for discovering the most suitable packets for dropping and forwarding. All incoming packet's stores according to the index of the packets mark by weight values (which is utilized to pick the next packet to transmit when a space is available) in every queue. The weight rule is to offer weight to the packets and divide them into Higher weight list (HWL) and low weight list (LWL) of packets. The packets that have low weight are schedule first and high weight HWL will be schedule if no low weight packets are available in LWL, otherwise drop the high weight noted packets. Different parameters are used for weight calculation. The proposed scheduled and drop policy dynamically calculates. The Fig. 3 shows the weight based drop and schedule architecture. The weight of each packet is calculated from the following Parameters.

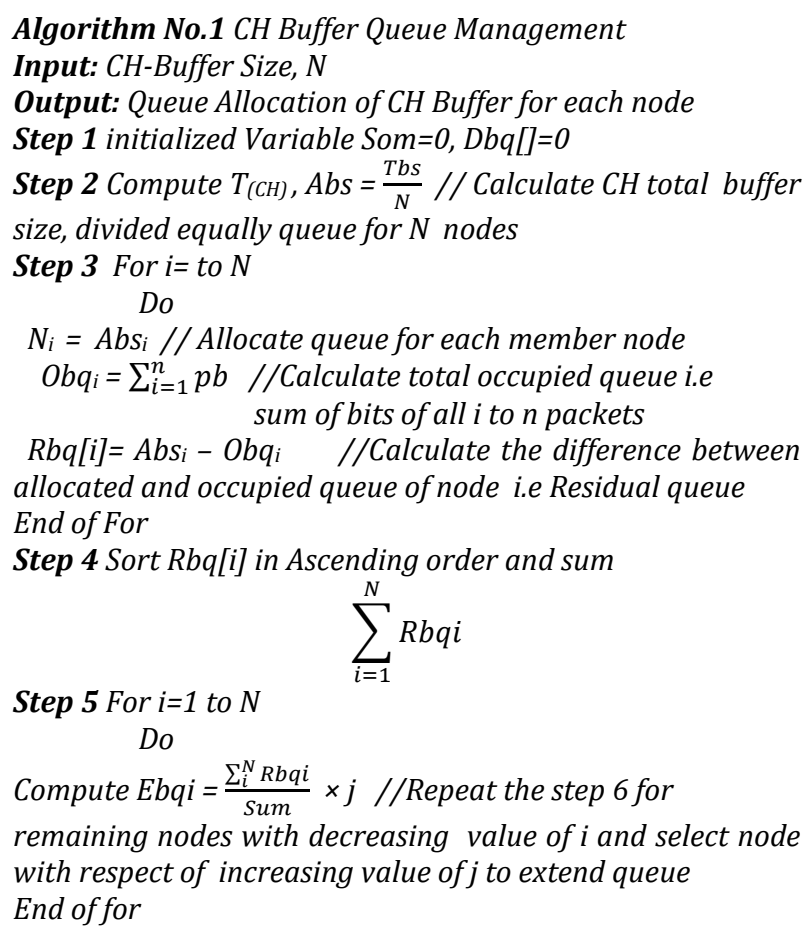

Table 1: CH Buffer Queue allocation before Queue management algorithm and packets receiving

\begin{tabular}{cccccc}
\hline Node ID & Allocated Queue & Received Packet in Size & Residual Queue (Rbs) & Required Queue & Extend Queue \\
\hline N-1 & $2500 \mathrm{~B}$ & $2000 \mathrm{~B}$ & $500 \mathrm{~B}$ & Nil & Nil \\
N-2 & $2500 \mathrm{~B}$ & $2800 \mathrm{~B}$ & Nil & $300 \mathrm{~B}$ & --- \\
N-3 & $2500 \mathrm{~B}$ & $2200 \mathrm{~B}$ & $300 \mathrm{~B}$ & $\mathrm{Nil}$ & $\mathrm{Nil}$ \\
N-4 & $2500 \mathrm{~B}$ & $2900 \mathrm{~B}$ & $\mathrm{Nil}$ & $400 \mathrm{~B}$ & --- \\
\hline
\end{tabular}

Table 2: CH Buffer Queue allocation after queue management algorithm

\begin{tabular}{cccccc}
\hline Node ID & Allocated Queue & Reveived Packet in Size & Residual Queue (Rbs) & Required Queue & Extend Queue \\
$\mathrm{N}-1$ & $2200 \mathrm{~B}$ & $2100 \mathrm{~B}$ & $100 \mathrm{~B}$ & Nil & Nil \\
$\mathrm{N}-2$ & $2700 \mathrm{~B}$ & $2800 \mathrm{~B}$ & $\mathrm{Nil}$ & $100 \mathrm{~B}$ & $200 \mathrm{~B}$ \\
$\mathrm{~N}-3$ & $2300 \mathrm{~B}$ & $2200 \mathrm{~B}$ & $100 \mathrm{~B}$ & $\mathrm{Nil}$ & $\mathrm{Nil}$ \\
$\mathrm{N}-4$ & $2800 \mathrm{~B}$ & $2900 \mathrm{~B}$ & $\mathrm{Nil}$ & $100 \mathrm{~B}$ & $300 \mathrm{~B}$ \\
\hline
\end{tabular}

\subsubsection{Remaining time to live (RTTL)}

Remaining Time to live is the imperative feature of the packet since it offers a sign that whether the packet is terminated or close to lapse and whether it is doable to repeat or not. This is very critical to decided RTTL on WMSN because of its temperament of disruption. Because of wireless nature some time packet takes longer time and expired before reaching to the destination.

\subsubsection{Size of packet (SF)}

The size of packet plays an imperative role in the buffer queue occupancy. The smaller size of packet enables $\mathrm{CH}$ to store more packets then larger size packet.

\subsubsection{Power of received signal strength of packet (RSSP)}

The received signal strength of particular packet plays an important role in buffer queue occupancy. RSSP can be considered as an indication for the link quality and the distance between nodes. The RSSI [13] is a function of the distance between two nodes and can be calculated as follows is Eq. 4 .

$\operatorname{RSSPi}(\mathrm{d})=\operatorname{RSSP}(\mathrm{d} 0)-10 \mathrm{n} \log \left(\frac{\mathrm{d}}{\mathrm{d} 0}\right)$

Where RSSPi(d) is the received signal strength in at a distance $d$ from the source node. RSSPi(d0) is the received signal strength at a distance $d 0$ from the source and $n$ is the attenuation exponent.

\subsubsection{Replication of packet (RC)}

This imply packet have replica if yes then how number of specific packet effectively got at $\mathrm{CH}$. Ought to this packet be discarded of, the assets already utilized are squandered. By decreasing HC packet loss rates, the squandering of system assets and influence utilization can be moved forward.

\subsubsection{Hop count (HC)}

Hop count is important for packet to be transported toward target nodes. Decreasing in the 
Hop counts toward destination is usually required because wireless transmissions are costly in terms of battery power and buffer.

\subsubsection{Packet delay (DFi)}

Delay is the difference in time between transmission from source and arrival at the $\mathrm{CH}$. Various statistics such as maximum delay, minimum delay and the variance of the delay are used when the describing the delay characteristics a given scheduling algorithm exhibits. The average delay of the i'th packet flow can be expressed by Eq. 5:

$\mathrm{DFi}=[\mathrm{rti}(\mathrm{j})-\operatorname{cti}(\mathrm{j})]$

where $\operatorname{cti}(\mathrm{j})$ is the time when the $\mathrm{j}$ 'th packet of the $i^{\prime}$ th node was transmitted from its source, cti(j) is the time when the j'th packet of the i'th node was received at the $\mathrm{CH}$.

The Eq. 6 shows the packet weight calculation using the above parameters.

$\mathrm{Wi}(\mathrm{f})=\mathrm{RTTLi}+\mathrm{SFi}+\mathrm{RSSPi}+\mathrm{RCi}+\mathrm{HCi}+\mathrm{DFi}$

\subsection{Operation of packet scheduling algorithm for queue}

When the queue size is full for particular node data and no further queue extension allowed by the queue extension algorithm 1 , then packet scheduling algorithm 1 start working to drop those packet for queue which high weight. First of all, Packet scheduling algorithm checks the availability of the queue when new incoming packets arrives at the queue. If queue have free space to hold new packet then add this packet to LWL list. If free space is not available for packet in the queue, then packet scheduling algorithm check two possibilities. Firstly, if the incoming packet not destine to the $\mathrm{CH}$ because of broadcast nature of every packet in WMSN, then drop it. Secondly if packet destine to $\mathrm{CH}$ for forwarding toward base station, then packet must be accommodated in the allocated queue by dropping some packets. For this, Packet scheduling algorithm is categorized packets according to the weight using Eq. 5. The low weight (LWL) packet marked not drop and high weight (HWL) marked drop. The packets from HWL are dropping until queue has enough space for the new arrival packet and terminate. If after deleting all packets form HWL but need more space for new arrival, then dropped packets from $\mathrm{LWL}$, so that the new incoming packets are accommodated. The Fig. 4 shows the packet scheduling algorithm. Table 3 show the packets weight calculation and division in two lists.

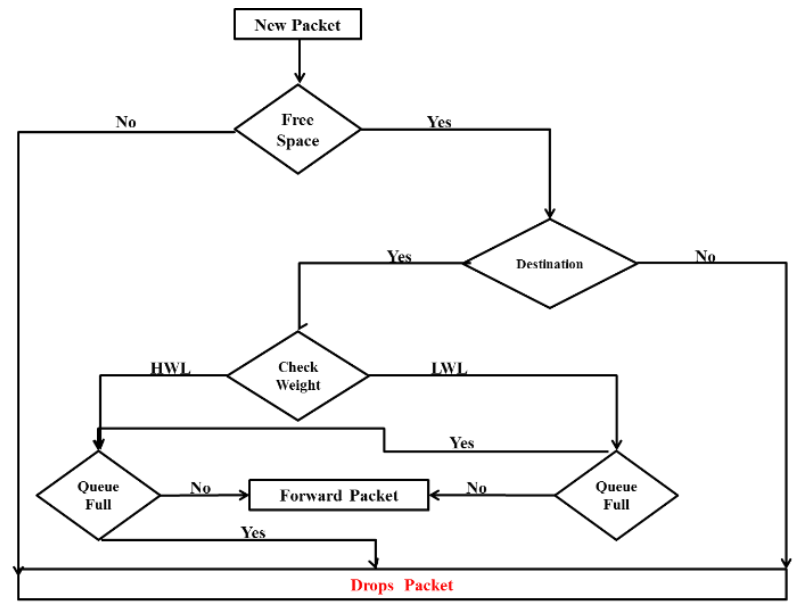

Fig. 3: Flow chart of packet scheduling

Table 3: Packets weight calculation for one node allocated queue at $\mathrm{CH}$ buffer

\begin{tabular}{cccccccc} 
Packets-ID & RTTL & SF & RSSP & RC & HC & DF & Weight \\
\hline P11 & 6 & 40B & 6 & 2 & 3 & 3 & 60 \\
P12 & 4 & 42B & 8 & 2 & 4 & 3 & 63 \\
P12 & 5 & 42B & 8 & 2 & 4 & 3 & 57 \\
P14 & 3 & 38B & 7 & 1 & 2 & 2 & 53 \\
P15 & 5 & 35B & 5 & 3 & 2 & 4 & 54 \\
P16 & 7 & 39B & 7 & 4 & 3 & 2 & 62 \\
P17 & 5 & 35B & 4 & 3 & 1 & 4 & 52 \\
P15 & 5 & 36B & 5 & 3 & 2 & 4 & 55
\end{tabular}

\section{Performance analysis}

The proposed solution has been validated through simulation and comparing its performance with the FCFS, DMP, EDP and Proposed algorithms. The proposed scheme aims to preserve as less energy as possible by selecting stable cluster and increases QoS. To compare the performance among FCFS, DMP, and EDP packet scheduling algorithm, we assume that all packets have the same size. The result comparison among proposed scheme and FCFS, DMP, EDP has been carried out using the following simulation parameters shown in Table 4 .

Network problems such as channel congestion and overflowed buffers can cause packet drops. Packet drop is defined as the difference between which the sink receives packets to the total packets sent via $\mathrm{CH}$.

Table 4: Simulation parameters

\begin{tabular}{cc}
\hline Parameter & Value \\
\hline Mobility model & Random way point \\
Number of sensor nodes & 100 \\
Length of Data packet & 50 Bytes \\
Length of control packet & 5 Bytes \\
Initial Energy & 1 Joule \\
Interface Queue type & FCFS, DMP, EDP, proposed \\
Communication model & scheme \\
Simulation Area & Bi-directional \\
Node Speed & $1000 \times 1000 \mathrm{~m} 2$ \\
Maximum Queue & $1-10$ m/second \\
Simulation Time & 50 packets \\
\end{tabular}


The proposed algorithm first provide queue extension to those nodes allocated queue in $\mathrm{CH}$ buffer, that generates large number of packets then usually node in network. Furthermore, if no further queue extension is allowed for these specific nodes, then packet scheduling algorithm drop those packet which have low weight. Therefore, congestion is managed properly and the receiving packet rate is improved at sink. Fig. 4 depicts the packet which is drops in the network using proposed scheme compared with three other schemes. It is clear from that the proposed scheme outperform when consider the packets drop. The dynamic queue allocation enables $\mathrm{CH}$ to provide dynamic queue in proportion to the number of packets a node generates in cluster. The weight base dropping police provides reduction in dropped packet. Weight controlled the massive drop of packets and enhanced delivery.

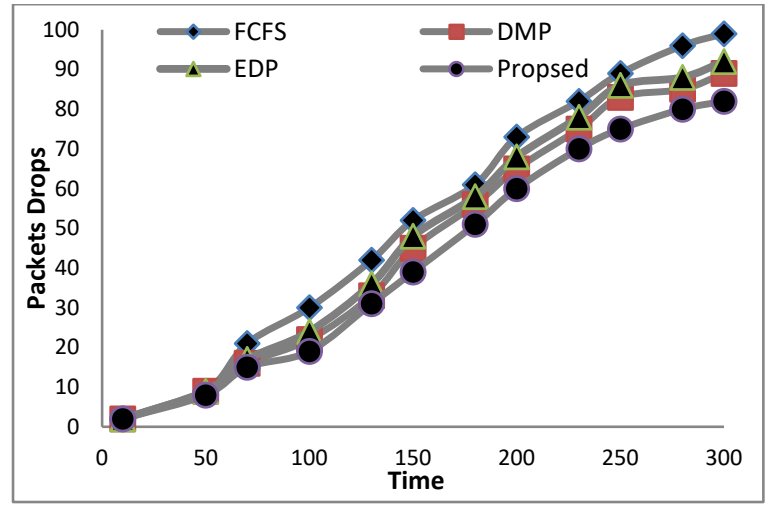

Fig. 4: Packets drops in specific time

In cluster topology, end-to-end delay depends on relative location between $\mathrm{CH}$ and $\mathrm{BS}$, and the buffer queue allocation of CHs as well. Increases in end-toend delay is very unavoidable when the quantity of packets increases in the cluster, since the proposed scheme uses dynamic queue allocation and scheduling for $\mathrm{CH}$.

Fig. 5 shows illustrate the total delay taken by the network according to the data traffic over a simulation time. By analyzing the result it is clear that proposed scheme decrease the delay time by assigning dynamic queue extension and weight based packets dropping. In weight calculation the packet delay parameters used, so those packet which have high delay time are dropped earlier. Packets drop and packet delivery ratio is inversely proportional.

This represents the difference ratio between the numbers of sent packets of network and the number of packets that are received by the base station. The proposed algorithm efficiently mange's $\mathrm{CH}$ buffer through an active queue management strategy by assigning dynamic buffer space to all neighboring nodes in proportion to the number of packets received from it and hence controlling packet drop probabilities. As a result the dropping ratio of the proposed scheme increases and the Fig. 6 shows the comparison between the proposed scheme and rest of schemes in term of packets delivery ratio. Fig. 7 shows the comparison through put obtaining using proposed, DMP, EDP and FCFS schemes. This shows that the proposed scheme obtain better throughout as compare to rest schemes because of $\mathrm{CH}$ dynamic queue management, weight based dropping and scheduling using different parameters.

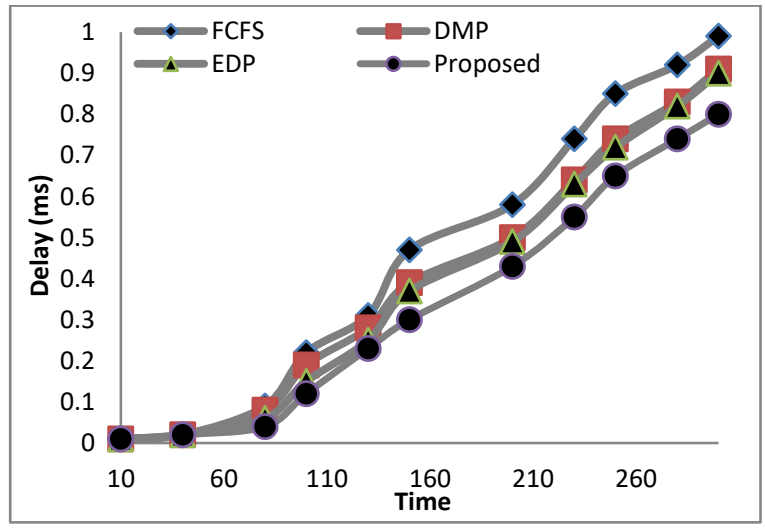

Fig. 5: Delay (ms) VS time

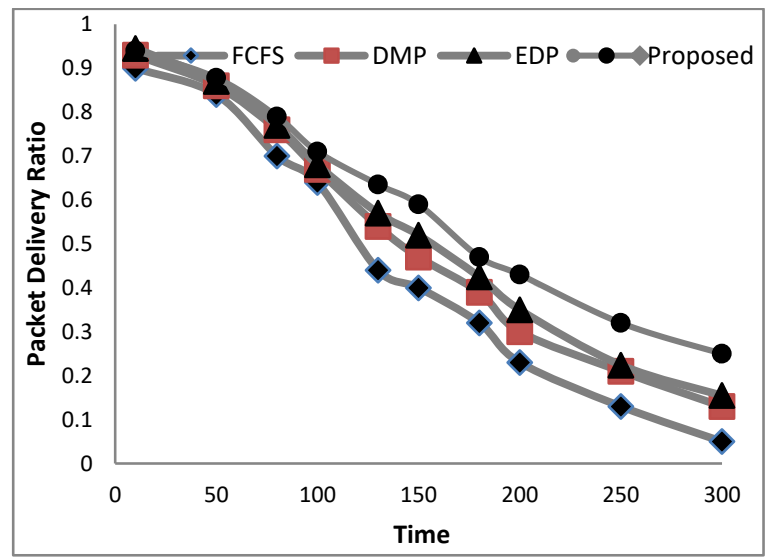

Fig. 6: Packets delivery ratio in time

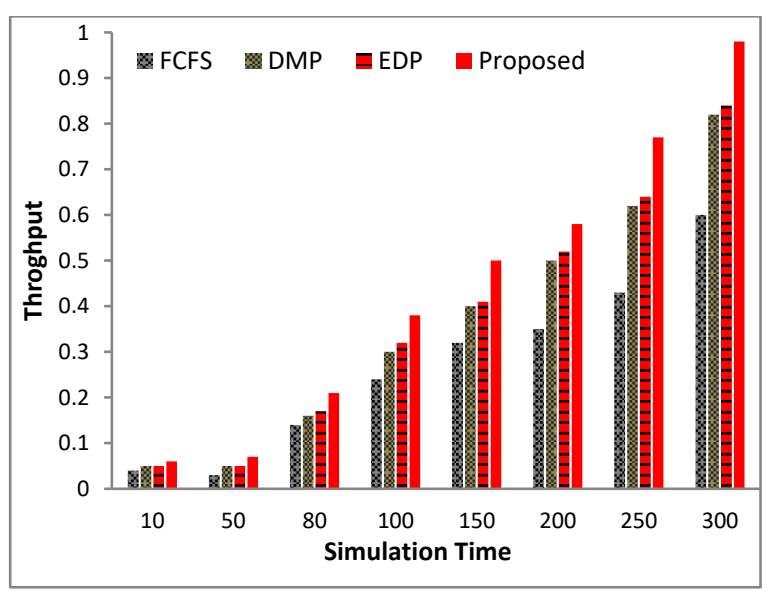

Fig. 7: Throughput of network in time

The average energy consumption ratio of the entire topology is the average difference between the initial level of energy and the final level of energy that is left in network. This metric is an important because the energy level of the network uses is proportional to the networks lifetime. The lower the energy consumption ratio the longer is the networks lifespan. Fig. 9 shows energy consumption ratio comparison of proposed scheme with other schemes. 
From this graph, it can be observed that the energy consumption ratio of proposed scheme is less than from the rest of three schemes.

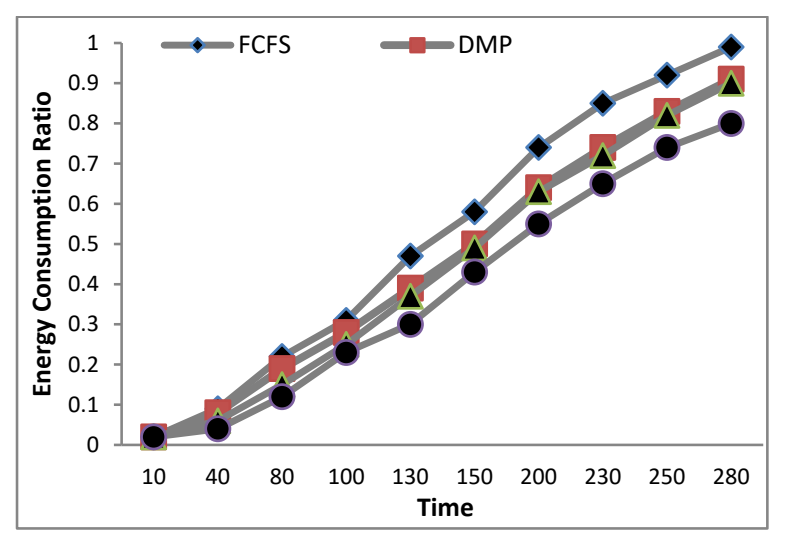

Fig. 9: Energy consumption ratio in time

\section{Conclusion}

This paper has presented buffer queue management and packet scheduling algorithm in WMSN. Buffer queue management algorithm has been presented for $\mathrm{CH}$ buffer management to enhance QoS by assigning dynamic buffer queue space of $\mathrm{CH}$ buffer to all member nodes in proportion to the number of packets received from member nodes and hence increase packet delivery ratio. Furthermore, packet scheduling algorithm enhanced packet delivery ratio by dropping and scheduling packets using weight and dynamic queue management. Each $\mathrm{CH}$ has made appropriated queue management and packet scheduling with high rate adjustment to realize whole cluster traffic.

\section{References}

Al-Karaki JN and Kamal AE (2004). Routing techniques in wireless sensor networks: a survey. IEEE Wireless Communications, 11(6): 6-28.

Bansode S and Sambare S (2015). Performance evaluation of dynamic multilevel priority (DMP) packet scheduling method for wireless sensor networks (WSNs). In the International Conference on Pervasive Computing, IEEE, Pune, India: 1-6. https://doi.org/10.1109/PERVASIVE.2015.7086986

Chipara O, Lu C, and Roman GC (2013). Real-time query scheduling for wireless sensor networks. IEEE Transactions on Computers, 62(9): 1850-1865.

Floyd S and Jacobson V (1993). Random early detection gateways for congestion avoidance. IEEE/ACM Transactions on Networking (ToN), 1(4): 397-413.

Gomathi R and Mahendran N (2015). An efficient data packet scheduling schemes in wireless sensor networks. In the $2^{\text {nd }}$ International Conference on Electronics and Communication Systems, IEEE, Coimbatore, India: 542-547. https://doi.org/ 10.1109/ECS.2015.7124966
Hu X and Guo W (2016). An efficient and stable congestion control scheme with neighbor feedback for cluster wireless sensor networks. KSII Transactions on Internet and Information Systems (TIIS), 10(9): 4342-4366.

Jiang C, Yuan D, and Zhao Y (2009). Towards clustering algorithms in wireless sensor networks-a survey. In the Wireless Communications and Networking Conference, IEEE, Budapest, Hungary: 1-6. https://doi.org/10.1109/WCNC.2009.4917996

Justus JJ and Sekar AC (2016). Energy efficient priority packet scheduling with delay and loss constraints for wireless sensor networks. In the International Conference on Inventive Computation Technologies, IEEE, Coimbatore, India, 3: 1-7. https://doi.org/10.1109/INVENTIVE.2016.7830076

Li Q, Li J, Wang J, and Sun W (2013). A dynamic cross-layer data queue management approach based on priority for delaytolerant mobile sensor networks. Journal of Electronics (China), 30(4): 328-334.

Lin QM, Wang RC, Jian GUO, and Sun LJ (2011). Novel congestion control approach in wireless multimedia sensor networks. The Journal of China Universities of Posts and Telecommunications, 18(2): 1-8.

Liu CM, Lee CH, and Wang LC (2007). Distributed clustering algorithms for data-gathering in wireless mobile sensor networks. Journal of Parallel and Distributed Computing, 67(11): 1187-1200.

Mitra R and Nandy D (2012). A survey on clustering techniques for wireless sensor network. International Journal of Research in Computer Science, 2(4): 51-57.

Nasser N, Karim L, and Taleb T (2013). Dynamic multilevel priority packet scheduling scheme for wireless sensor network. IEEE Transactions on Wireless Communications, 12(4): 1448-1459.

Periyasamy R and Perumal D (2015). A game theory-based hybrid medium access control protocol for congestion control in wireless sensor networks. In: Hausken K and Zhuang J (Eds.), Game theoretic analysis of congestion, safety and security: 125. Springer, Berlin, Germany.

Rehman E, Sher M, Hussnain S, and Ghani A (2016). Enhancing energy efficiency and qos using buffer queue management algorithms in stable clustering for wireless mobile sensor network. International Journal of Computer Science and Information Security, 14(11): 714-722.

Shuaib AH and Aghvami AH (2009). A routing scheme for the IEEE-802.15. 4-enabled wireless sensor networks. IEEE Transactions on Vehicular Technology, 58(9): 5135-5151.

Tsai TY, Chung YL, and Tsai Z (2010). Introduction to packet scheduling algorithms for communication networks. In Communications and Networking. InTech. Available online at: https://www.intechopen.com

Vanithamani S and Mahendran N (2014). Performance analysis of queue based scheduling schemes in wireless sensor networks. In the International Conference on Electronics and Communication Systems, IEEE, Coimbatore, India: 1-6. https://doi.org/10.1109/ECS.2014.6892593

Yantong W and Sheng Z (2016). An enhanced dynamic priority packet scheduling algorithm in wireless sensor networks. In the $18^{\text {th }}$ International Conference on Computer Modelling and Simulation, IEEE, Cambridge, UK: 311-316. https://doi.org/ 10.1109/UKSim.2016.31 